Article

\title{
Tyrosol and Hydroxytyrosol Determination in Extra Virgin Olive Oil with Direct Liquid Electron Ionization-Tandem Mass Spectrometry
}

\author{
Veronica Termopoli ${ }^{1}$, Maurizio Piergiovanni ${ }^{1}\left(\mathbb{D}\right.$, Achille Cappiello ${ }^{1,2}$, Pierangela Palma ${ }^{1,2}$ \\ and Giorgio Famiglini ${ }^{1, *(D)}$ \\ 1 LC-MS Laboratory, Department of Pure and Applied Sciences, University of Urbino Carlo Bo, \\ 61029 Urbino, Italy; veronica.termopoli@uniurb.it (V.T.); m.piergiovanni1@campus.uniurb.it (M.P.); \\ achille.cappiello@uniurb.it (A.C.); pierangela.palma@uniurb.it (P.P.) \\ 2 Department of Chemistry, Vancouver Island University (VIU), Nanaimo, BC V9R 5S5, Canada \\ * Correspondence: giorgio.famiglini@uniurb.it
}

\section{check for} updates

Citation: Termopoli, V.; Piergiovanni, M.; Cappiello, A.; Palma, P.;

Famiglini, G. Tyrosol and Hydroxytyrosol Determination in Extra Virgin Olive Oil with Direct Liquid Electron Ionization-Tandem Mass Spectrometry. Separations 2021, 8, 173. https://doi.org/10.3390/ separations 8100173

Academic Editors: Markus Ganzera and Didier Thiébaut

Received: 22 July 2021

Accepted: 29 September 2021

Published: 6 October 2021

Publisher's Note: MDPI stays neutral with regard to jurisdictional claims in published maps and institutional affiliations.

Copyright: (c) 2021 by the authors. Licensee MDPI, Basel, Switzerland. This article is an open access article distributed under the terms and conditions of the Creative Commons Attribution (CC BY) license (https:// creativecommons.org/licenses/by/ $4.0 /)$.

\begin{abstract}
Extra virgin olive oil (EVOO) is one of the main ingredients of the Mediterranean diet. It is claimed as a functional food for its unique content of health-promoting compounds. Tyrosol (Tyr), Hydroxytyrosol (Htyr), and their phenolic derivatives present in EVOO show beneficial properties, and their identification and quantification, both in their free form and after the hydrolysis of more complex precursors, are important to certify its quality. An alternative method for quantifying free and total Tyr and Htyr in EVOO is presented using an LC-MS interface based on electron ionization (EI), called liquid electron ionization (LEI). This method requires neither sample preparation nor chromatography; the sample is diluted and injected. The selectivity and sensitivity were assessed in multiple reaction monitoring mode (MRM), obtaining confirmation and quantification in actual samples ranging from 5 to $11 \mathrm{mg} / \mathrm{Kg}$ for the free forms and from 32 to $80 \mathrm{mg} / \mathrm{Kg}$ for their total amount after hydrolysis. Two MS/MS transitions were acquired for both compounds using the $\mathrm{Q} / \mathrm{q}$ ratios as confirmatory parameters. Standard addition calibration curves demonstrated optimal linearity and negligible matrix effects, allowing a correct quantification even without expensive and difficult to find labeled internal standards. After several weeks of operation, the system's repeatability was excellent, with an intraday RSD (\%) spanning from five to nine and an interday RSD (\%) spanning from 9 to 11.
\end{abstract}

Keywords: extra virgin olive oil (EVOO); liquid electron ionization (LEI); LC-MS; tyrosol; hydroxytyrosol

\section{Introduction}

Extra virgin olive oil (EVOO) is widely used in the Mediterranean diet. EVOO is obtained after mechanical treatments of olives aimed to preserve their nutritional characteristics, and it is gaining interest worldwide thanks to its well-known dietary and nutraceutical values associated with several health benefits [1-4]. It is characterized by a unique composition of minor components, together with many monounsaturated fatty acids, triacylglycerols, vitamin K, and vitamin E. Minor components are phenolic compounds belonging to four major classes: flavonoids, lignans, simple phenols, and secoiridoids [5]. Polyphenols in olives are unique and responsible for their organoleptic and sensory properties, such as the characteristic and distinctive aroma [6]. Polyphenols may inhibit oxidation reactions of EVOO and indirectly play a significant role in preventing cancer, aging, and chronic diseases, such as atherosclerosis, obesity, diabetes, and many others. [7-15].

Several parameters contribute to the presence of phenolic compounds and their derivatives in EVOO, such as cultivar, fruit integrity and maturity, agricultural practices, production processes, and storage length. In the literature of the last ten years, many studies aimed to optimize all of those parameters that can improve the qualitative and 
quantitative composition of the phenolic profile. Several other studies have attempted to elucidate the ultimate mechanisms through which EVOO-derived phenols contribute to health benefits. Among all the phenols, hydroxytyrosol (Htyr) was listed as one of the most potent therapeutic and nutraceutical agents for its antimicrobial, antithrombotic, and anti-inflammatory effects and its capacity to eradicate intracellular and extracellular reactive oxygen species $[16,17]$.

European Commission (EC) Regulation No. 432/2012 has listed the permitted health claims for foods and nutrients that refer to the reduction of disease risk and children's development and health [18]. Olive oil polyphenols are claimed to "contribute to the protection of blood lipids from oxidative stress", and "the claim may be used only for olive oil containing at least $5 \mathrm{mg}$ of hydroxytyrosol and its derivatives (e.g., oleuropein complex and tyrosol, Tyr) per $20 \mathrm{~g}$ of olive oil. In order to bear the claim, information shall be given to the consumer that the beneficial effect is obtained with a daily intake of $20 \mathrm{~g}$ of olive oil" [19].

Starting from this regulation, the EC encourages EVOO producers to dose polyphenols in their products to certify high-quality and healthy properties. A clear, descriptive label on the bottles should inform consumers of the potential nutritional benefits. Despite this suggestion, the regulation does not indicate the official methods to obtain these data, and, at present, only a limited number of EVOO products are labeled with this information. This gap lies in the difficulty of determining the polyphenols content accurately and the lack of official methods and a list of specific molecules to be determined. Most of the recommended methods have been proposed by the International Olive Council (IOC).

In 2009, the IOC approved a method in which polyphenols are extracted with a hydroalcoholic solution and analyzed by HPLC-DAD [20]. Even though the detection and quantification of all the phenolic substances, including flavonoids, is demonstrated, retention times and UV absorbance at $280 \mathrm{~nm}$ might not be sufficient to exclude false positives or erroneous quantification. Olive oil composition was explored in many scientific publications, and different protocols were presented. None of these methods can be considered "official" because no new regulations have been issued to fulfill this purpose [21,22]. GC-FID and LC-DAD are commonly used because they are relatively inexpensive, straightforward, readily available, and suitable with quality control laboratory practices [20,22-27]. The LC-MS and LC-MS/MS methods enhance selectivity and sensitivity in combination with complex and time-consuming sample preparation steps, such as liquid-liquid extraction (LLE), solid-phase extraction (SPE), or derivatization protocols. In most cases, sample preparation involves a hydrolysis step where all the polyphenols are reduced to simple phenols (Tyr and Htyr) to quantify their total content either in the polar fraction or directly in oil or olive oil mill wastewaters [28-33]. However, Tyr and Htyr are simple phenols to dose in oil samples to comply with EC regulation N. 432/2012, either in their free form or after the hydrolysis of more complex phenols. Simplified analytical protocols and confirmatory techniques are invoked to fulfill the lack of official methods for olive oil certification in terms of quality and nutritional properties [34]. Recently, paper spray tandem mass spectrometry was applied to quantify free and total Tyr and Htyr with good results in terms of reliability, rapidity, and sensitivity [35]. Alternatively, EVOO was characterized by the direct determination of the secoiridoids without the hydrolysis of the sample [36].

In this work, we present an alternative method to quantify free and total Tyr and Htyr in EVOO samples using a liquid electron ionization (LEI-MS/MS) approach in flow injection analysis (FIA) mode. LEI is a new LC-MS interface able to vaporize a nano-flow liquid effluent at ambient pressure before entering into an electron ionization MS source. One of the peculiar advantages of the LEI interface is the relatively low matrix effects (ME), which guarantees quantitative results from over- or underestimations [37,38]. A chromatographic pump conveys the samples into the LEI interface, where they vaporize before entering the EI source. Compared to previous attempts, the proposed method does not require chromatographic separation due to MS/MS selectivity and LEI robustness. This approach can be used for the preliminary screening of oil samples to identify and 
quantify free Tyr and Htyr to rapidly certify the excellent quality of olive oils, bypassing the purification or pre-concentration steps and time-consuming sample preparations. Oil samples with a low concentration of free Tyr and Htyr should be investigated deeper, after polyphenols hydrolysis, before quantification. This method was applied to the analysis of Htyr and Tyr of three EVOO samples produced in Italy in different regions: Marche, Sardinia, and Apulia. High-molecular weight polyphenols that do not vaporize using the LEI interface were hydrolyzed before the analysis to determine the total Tyr and Htyr amount required for the health claim.

\section{Materials and Methods}

\subsection{Chemicals}

The analytical standards of 3-hydroxytyrosol (Htyr) (CAS 10597-60-1) and of 2-(4-hydroxyphenyl)ethanol (Tyr) (CAS 501-94-0) (purity $\geq 98 \%$ ) were purchased from Sigma-Aldrich (Milan, Italy). Water, acetonitrile, ethanol, and acetone (HyperSolv Chromanorm LC-MS grade) were purchased from VWR, Part of Avantor (Milan, Italy). $\mathrm{HCl}$ $(37 \%)$, and $\mathrm{HCOOH}(\geq 96 \%)$ was purchased from Sigma-Aldrich (Milan, Italy).

\subsection{Standard Solutions and Extra Virgin Olive Oil Samples}

Tyr and Htyr stock solutions were prepared gravimetrically at the concentration of 1 and $10 \mathrm{mg} / \mathrm{mL}$ in acetone. All solutions were stored at $4{ }^{\circ} \mathrm{C}$ in sealed amber glass vials. Working solutions of each compound were prepared daily by diluting the stock solutions with acetone.

EVOO samples $(n=3)$ from Apulia, Marche, and Sardinia regions were produced from local groves in 2019-2020 and stored in dark containers. Before determining the free analytes, each sample was weighted to obtain $100 \mathrm{mg}$ of sample and diluted 1:10 (w:v) with acetone before analysis.

EVOO samples were submitted to hydrolysis [30] to quantify Tyr and Htyr total amount. An aliquot $\left(500 \mathrm{mg}\right.$ ) of each sample was extracted with $1 \mathrm{~mL}$ of $\mathrm{EtOH} / \mathrm{H}_{2} \mathrm{O}$ $(0.1 \% \mathrm{HCOOH}) 7: 3(v: v)$ vortexing for $3 \mathrm{~min}$ and then centrifuged. $100 \mu \mathrm{L}$ of $2 \mathrm{M} \mathrm{HCl}$ was added to $100 \mu \mathrm{L}$ of the extract and hydrolyzed at $950 \mathrm{~W}$ microwave power at $130{ }^{\circ} \mathrm{C}$ for 10 min (MDS-2100-CEM-Microwave Technology Ltd., Buckingham, UK). The procedure was carried out in triplicate. Each hydrolysate was then diluted with $200 \mu \mathrm{L}$ of acetone before the analysis.

Five-point calibration curves were plotted using the standard addition method for each EVOO sample ( $\cong 100 \mu \mathrm{L}$ ) by adding $2,4,6,8 \mu \mathrm{L}$ of the $1 \mathrm{mg} / \mathrm{mL}$ standard solutions. The same procedure was used to construct five-point calibration curves to determine the total amount of Tyr and Htyr after the hydrolysis process for each EVOO sample. Amounts of 2, 4, 6, and $8 \mu \mathrm{L}$ of the $10 \mathrm{mg} / \mathrm{mL}$ standard solutions of Tyr and Htyr were added to the hydrolyzed samples.

After quantification (free and hydrolyzed Tyr and Htyr), fortified samples in acetone were prepared at the same concentration levels, and standard addition curves were plotted utilizing the procedure described above for matrix effect evaluation.

Free Tyr concentration determined in EVOO Marche and free Htyr concentration determined in EVOO Apulia were utilized to fortify corn oil samples, and standard addition curves were plotted starting from these fortified samples. Furthermore, standard addition calibration curves for corn oil were plotted from Tyr and Htyr concentrations determined in the hydrolyzed EVOO Marche sample. These corn oil curves helped to compare actual and acetone samples with a similar complex sample free of the analytes.

\subsection{LC-LEI-MS/MS Apparatus and Working Conditions}

An Agilent 1100 series nanoPump (Agilent Technologies, Palo Alto, CA, USA) was coupled to a triple quadrupole MS (Agilent 7010B, Agilent Technologies) operating in EI via an LEI interface and equipped with a high-efficiency source (HES) that allowed high ionization efficiency. The mobile phase was $20: 80 \mathrm{H}_{2} \mathrm{O}: \mathrm{ACN}(v: v)$ at a $400 \mathrm{~nL} / \mathrm{min}$ flow rate. 
A nano-column (Agilent Zorbax SB-C18, $0.075 \times 150 \mathrm{~mm}, 3.5 \mu \mathrm{m}$ particle size) was installed between the pump flow rate exit and the injector to guarantee sufficient backpressure to work in stable conditions and not for chromatographic purposes. Standard solutions and EVOO actual samples were analyzed in FIA using an external injector equipped with a $100 \mathrm{~nL}$ internal sample loop (VICI AG International, Schenkon, Switzerland). The LEI interface was thoroughly described elsewhere [36,37]; in this application, the vaporization micro-channel temperature was set at $300{ }^{\circ} \mathrm{C}$. MS tuning was performed weekly at an ion source temperature of $280^{\circ} \mathrm{C}$ using perfluorotributylamine as a reference compound. Check tune was performed daily in the same conditions. No mobile phase was admitted into the ion source during the tuning procedure. Analyses were carried out in full scan and multiple reaction monitoring (MRM) using the $\mathrm{m} / \mathrm{z}$ range and transitions reported in Table 1 . Ions at m/z 138 and 154 are radical ions formed during the EI process. They are used as precursor ions in MS/MS experiments. In Figure 1, structures and LEI-MS spectra of Tyr and Htyr are shown.

Table 1. Full scan, MS/MS parameters, and Q/q ratios (reference and experimental) of the selected phenolic compounds. $\mathrm{Q} / \mathrm{q}$ ratios were calculated as the average of five injections at five calibration levels (three replicates each); relative standard deviation for solvent (Reference) and diluted EVOO samples (Experimental) are reported in parentheses.

\begin{tabular}{|c|c|c|c|c|c|c|}
\hline Compound & $\begin{array}{l}\text { Quantitative } \\
\text { Transition (Q) }\end{array}$ & $\mathrm{CE}(\mathrm{eV})$ & $\begin{array}{c}\text { Qualitative } \\
\text { Transition (q) }\end{array}$ & $\mathrm{CE}(\mathrm{eV})$ & $\begin{array}{c}\text { Reference } \\
Q / q \pm \operatorname{RSD}(\%)\end{array}$ & $\begin{array}{l}\text { Experimental } \\
\mathrm{Q} / \mathrm{q} \pm \mathrm{RSD}(\%)\end{array}$ \\
\hline Tyr & $138 \rightarrow 107$ & 15 & $138 \rightarrow 77$ & 10 & $5.5(10)$ & $5.7(9)$ \\
\hline Htyr & $154 \rightarrow 123$ & 10 & $154 \rightarrow 77$ & 10 & $6.4(5)$ & $6.8(7)$ \\
\hline \multirow{2}{*}{\multicolumn{4}{|c|}{ Full scan }} & $\mathrm{m} / \mathrm{z}$ & Data sample & $\mathrm{Cyc} / \mathrm{s}$ \\
\hline & & & & $55-250$ & 700 & 1.4 \\
\hline
\end{tabular}
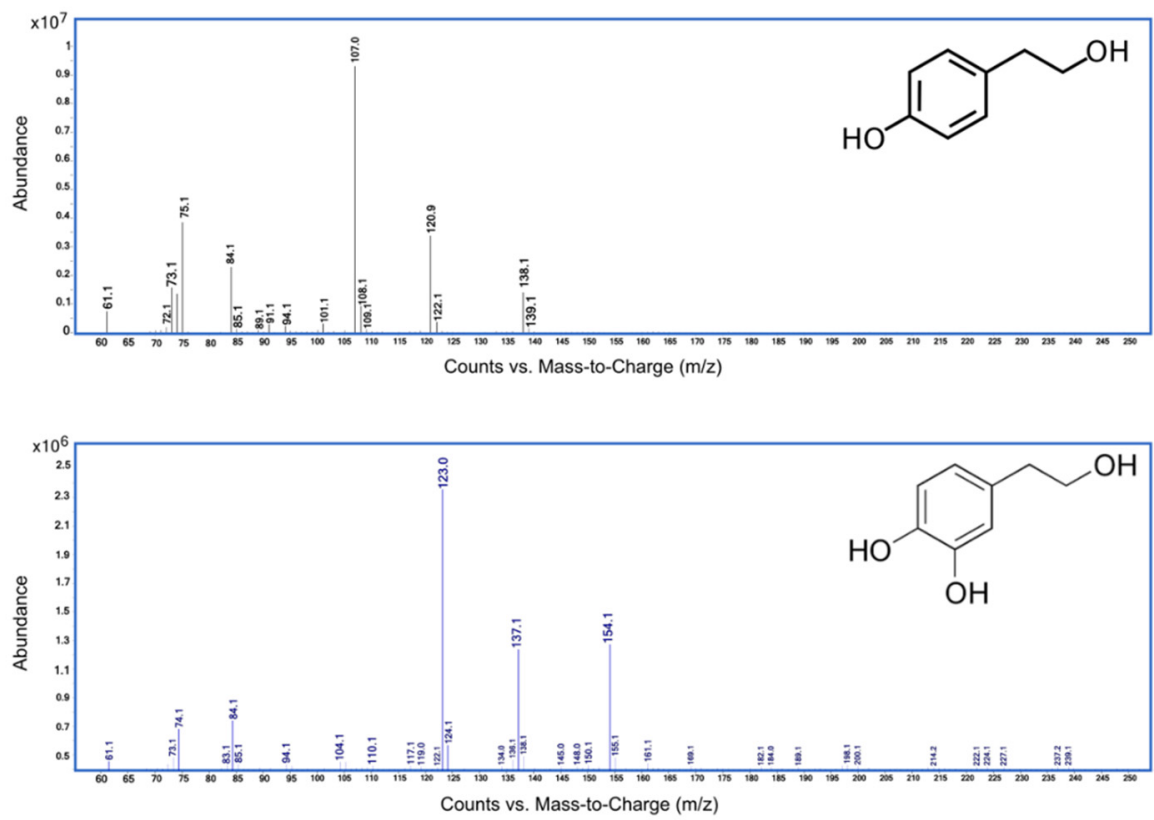

Figure 1. Structures and LEI-MS spectra of (a) Tyr and (b) Htyr.

\section{Results and Discussion}

LEI-MS/MS offers a valid alternative in analyzing free and hydrolyzed phenols in EVOO because it relies on EI, a gas-phase ionization technique that occurs in-source at a high temperature and high vacuum where ion-ion or ion-molecule reactions are infrequent. These conditions explain why matrix effects (ME) are limited or absent compared with other LC-MS approaches [38]. Methods based on ESI-MS can provide accurate quantita- 
tive results only after addressing ME issues. Coeluted matrix components can represent a significant limitation when no sample preparation and fast analysis are required. Timeconsuming and expensive extraction and purification procedures are needed to avoid or limit signal suppression or enhancement and develop adequate ME evaluation procedures. The EI process is highly reproducible, suitable for inter-laboratory assays, and the resulting spectra can be compared with those present in database libraries for the undoubted identification in targeted and untargeted analysis.

\subsection{Qualitative Results}

Qualitative identification in the target analysis of complex actual samples can benefit from the MS/MS approach increasing sensitivity and selectivity, even in the presence of high concentrations of interfering compounds. The $\mathrm{Q} / \mathrm{q}$ ratio was calculated to confirm the peak identity in actual and fortified samples. Experimental Q/q ratios obtained from the actual samples were compared with those obtained with standard solutions. The confirmation of the analytes in the EVOO samples was considered positive when the experimental Q/q ratio was within $\pm 20 \%$ of the average $Q / q$ value calculated from the standards [39]. In Table 1, the reference and experimental Q/q ratios are reported, confirming the presence of the target analytes in the actual samples. This comparison is fundamental for the undoubted analytes identification, especially in direct analysis without chromatographic separation. Blank specimens were analyzed to assess the presence of possible interferents.

\subsection{Method Validation}

The method was validated considering linearity $\left(\mathrm{R}^{2}\right)$, LODs, LOQs, intraday, interday precision, and ME evaluation.

\subsubsection{Linear Range}

Linearity was evaluated using the standard addition method, as described in Section 2.2. Calibration curves were plotted using the least-squares regression analysis of the signal intensity (peak area) versus the Tyr and Htyr absolute added amount. The linearity was evaluated from $X$ to $X+8 \mu \mathrm{g}$ for the determination of free Tyr and Htyr and from $X$ to $X+80 \mu \mathrm{g}$ for the compounds determination after hydrolysis. Each point on the curve is the mean of five replicates. The same experiments were conducted on the EVOO samples after hydrolysis to quantify the Tyr and Htyr total amount. The linear regression equations and Pearson square determination coefficients $\left(\mathrm{R}^{2}\right)$ obtained from the actual samples, solvent samples, and corn oil samples are reported in Table 2 . The $\mathrm{R}^{2}$ values are between 0.9942 and 0.9999 , demonstrating a good linearity given the high matrix complexity. Acetone and corn oil samples were used to evaluate the ME and compare the linear regression in a complex matrix without analytes. 
Table 2. Linear regression equations, LODs, and LOQs of free and hydrolyzed samples.

\begin{tabular}{|c|c|c|c|c|c|c|c|}
\hline \multirow{3}{*}{ Matrix } & \multirow{3}{*}{ Working Range ( $\mu \mathrm{g}$ ) } & \multicolumn{4}{|c|}{ Free } & \multirow{3}{*}{\multicolumn{2}{|c|}{ LODs (ng/mL) }} \\
\hline & & Tyr & \multirow{2}{*}{$R^{2}$} & Htyr & \multirow{2}{*}{$\mathbf{R}^{2}$} & & \\
\hline & & Equations & & Equations & & & \\
\hline Acetone & \multirow{5}{*}{$0-8$} & $y=2964 x-179.44$ & 0.9942 & - & 0.9957 & Tyr & Htyr \\
\hline EVOO Marche & & $y=2808.2 x-167.4$ & 0.9981 & n.d & - & 10 & 30 \\
\hline Corn oil & & $y=2924.2 x-171.4$ & 0.9974 & $y=2282 x+89.24$ & 0.9984 & \multicolumn{2}{|c|}{ LOQs (ng/mL) } \\
\hline Acetone & & $y=3324.7 x-315.64$ & 0.9922 & $y=2337.4 x+134.28$ & 0.9957 & Tyr & Htyr \\
\hline EVOO Apulia & & $y=3293.6 x-288.72$ & 0.9910 & $y=2235 x+119.6$ & 0.9997 & 50 & 70 \\
\hline \multicolumn{8}{|c|}{ Hydrolyzed } \\
\hline Acetone & & $y=1968.5 x+8385.8$ & 0.9993 & $y=1145.6 x+2605.1$ & 0.9994 & & \\
\hline EVOO Marche & \multirow{6}{*}{$0-80$} & $y=1925.3 x+8121.1$ & 0.9995 & $y=1136.4 x+2302.4$ & 0.9997 & & \\
\hline Corn oil & & $y=1959.8 x+8241.7$ & 0.9978 & $y=1132.4 x+2285.3$ & 0.9998 & & \\
\hline Acetone & & $y=2337.2 x+11639$ & 0.9991 & $y=1636.7 x+4617.8$ & 0.9999 & & \\
\hline EVOO Apulia & & $y=2309.7 x+11540$ & 0.9992 & $y=1615.9 x+4406.3$ & 0.9999 & & \\
\hline Acetone & & $y=1591.8 x+6979.6$ & 0.9998 & $y=1446.8 x+3336.1$ & 0.9984 & & \\
\hline EVOO Sardinia & & $y=1537.3 x+6593.1$ & 0.9991 & $y=1406 x+3053.9$ & 0.9986 & & \\
\hline
\end{tabular}

\subsubsection{Limits of Detection and Quantification}

The instrumental LOD was considered as the absolute amount of the compound, giving an $\mathrm{S} / \mathrm{N}$ of $3 / 1$, whereas the instrumental LOQ was set at an $\mathrm{S} / \mathrm{N}$ of 10/1, expressing the lowest amount of the analyte that can be determined quantitatively. The LODs and LOQs were calculated by injecting a standard solution of 20 and $100 \mathrm{ng} / \mathrm{mL}$ of Tyr and Htyr. All of the data are reported in Table 2. These values are comparable with those obtained with other methods using LC-UV or DAD detectors or, in some cases, LC-MS, demonstrating the robustness of the method [22,35].

\subsubsection{Evaluation of Matrix Effects}

Interfering compounds present in the matrix represent a possible drawback that must be addressed in quantitative MS analysis. These compounds can enhance or suppress the analyte's signal and cause misleading results. The LEI shows negligible ME, as already demonstrated [37,40]. Nevertheless, because EVOO is a complex matrix, a thorough evaluation of the ME is mandatory. The evaluation of the ME was made comparing the calibration curves of the Tyr and Htyr standards in acetone, corn oil, and EVOO (Figure 2). In ideal conditions, the total absence of ME is observed when the two slopes are identical. The slopes are overlapping, spanning from $95 \%$ to $97 \%$ for both compounds in all the EVOO samples (Table 3). The slight difference in the values can be ascribed to normal instrumental variations in LC-MS/MS experimental data acquisition. The ME evaluation demonstrates method robustness, especially considering that neither sample pretreatment nor chromatographic separation was performed. The corn oil overlapping value $(99 \%)$ for free and hydrolyzed Tyr and Htyr in the three samples confirms that matrix components do not interfere considerably with analytes quantification.

\subsubsection{Precision}

The intraday and interday precisions were evaluated by injecting nine replicates of a $100 \mathrm{mg}$ Marche EVOO sample at the concentration of $X+4 \mathrm{mg}$ of both compounds for five consecutive days. The results obtained show good repeatability: the intraday RSD spanned from $4.5 \%$ to $9.0 \%$ for Tyr and from 3\% to $7 \%$ for Htyr. The interday precision values spanned from $9.0 \%$ to $11.0 \%$ for Tyr and from $7.5 \%$ to $10.5 \%$ for Htyr. The precision data were not acquired for hydrolyzed samples. 


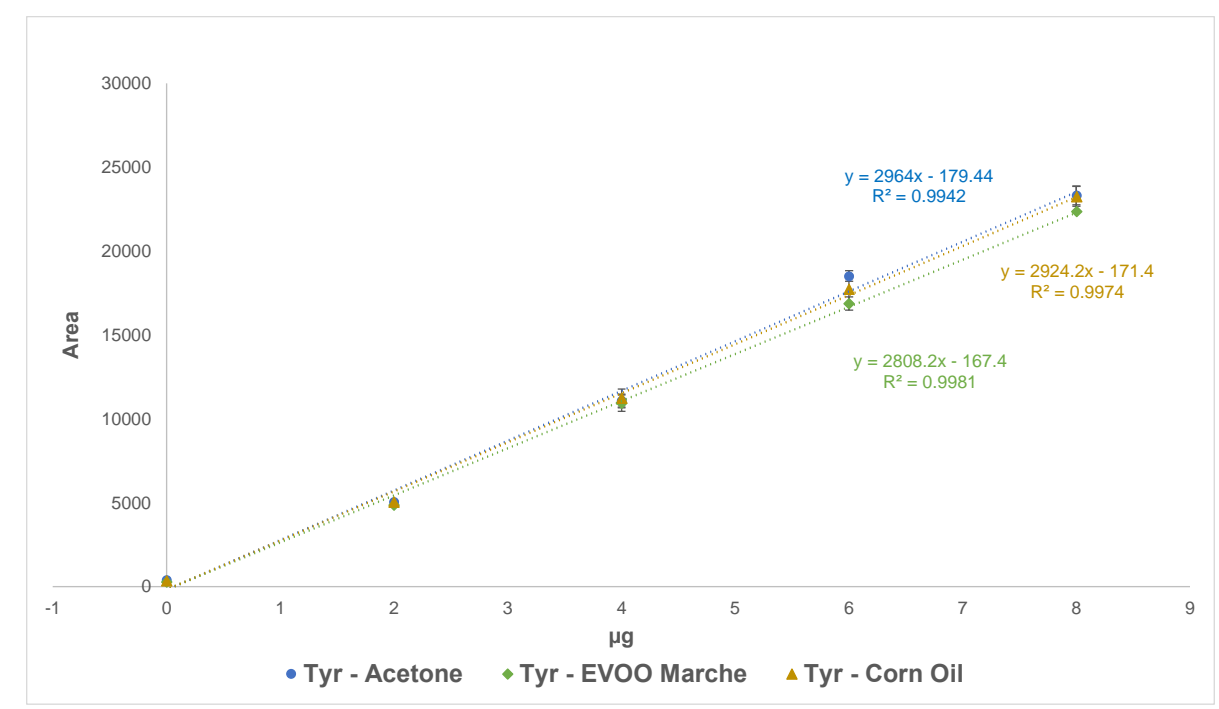

(a)

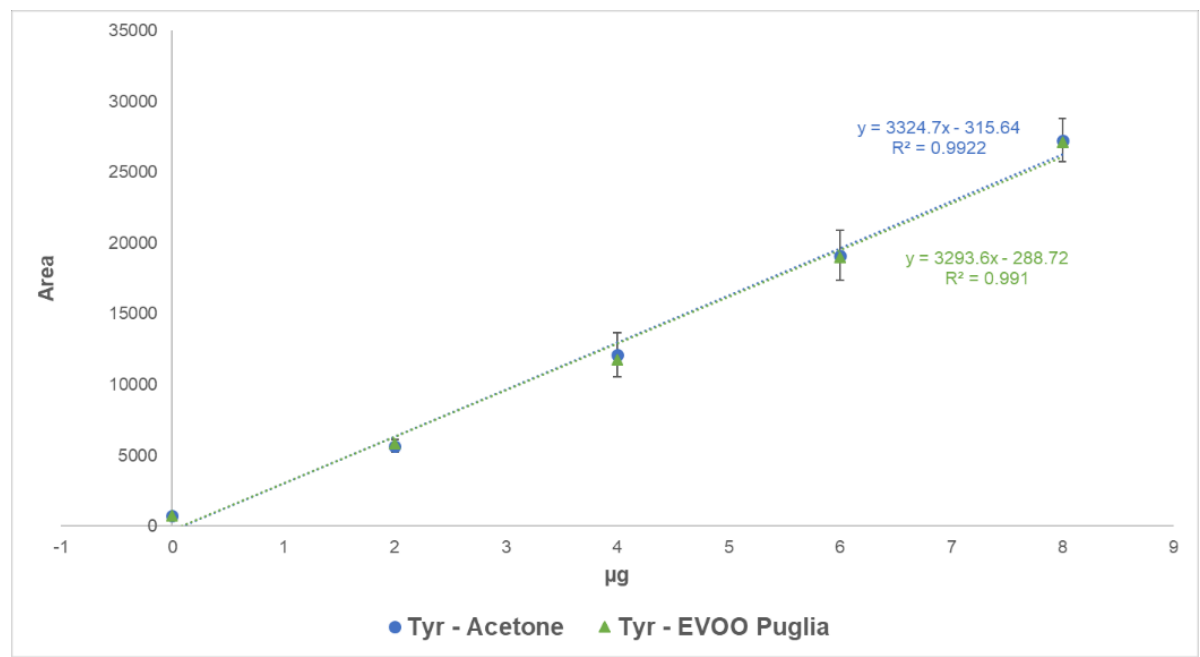

(b)

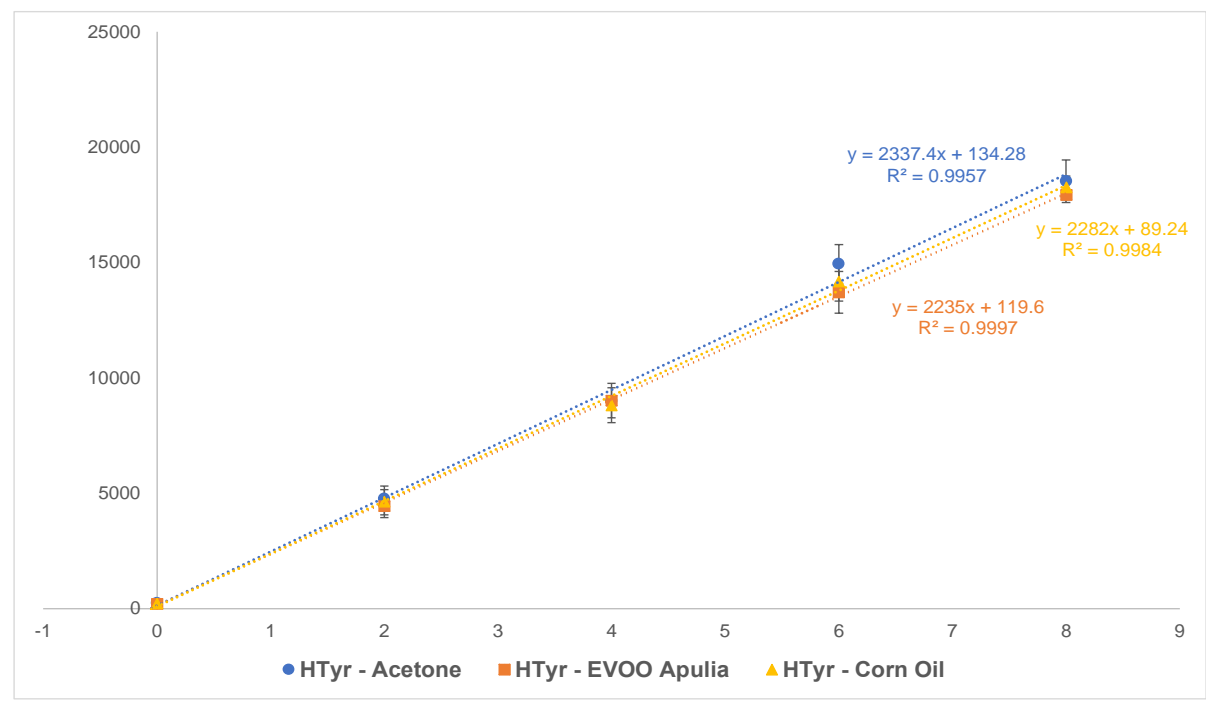

(c)

Figure 2. Matrix effect evaluation. Comparison of standard addition calibration curves: (a) Tyr in solvent (blue line), EVOO Marche (green line), and corn oil (yellow line); (b) Tyr in solvent (blue line) and EVOO Apulia (green line). (c) Htyr in solvent (blue line), EVOO Apulia (orange line), and corn oil (yellow line). 
Table 3. Matrix effects evaluation of the quantified EVOO samples.

\begin{tabular}{ccc}
\hline \multirow{2}{*}{ Compound } & \multicolumn{2}{c}{ Matrix Effects Evaluation (a) } \\
\hline \multirow{2}{*}{ Free Tyr } & EVOO Marche & $95 \%$ \\
\cline { 2 - 3 } & EVOO Apulia & $99 \%$ \\
\cline { 2 - 3 } Free Htyr & Corn oil & $99 \%$ \\
\cline { 2 - 3 } & EVOO Apulia & $96 \%$ \\
\cline { 2 - 3 } Hydrolyzed Tyr & Corn oil & $97 \%$ \\
\cline { 2 - 3 } & EVOO Marche & $98 \%$ \\
\hline \multirow{2}{*}{ Hydrolyzed Htyr } & EVOO Apulia & $99 \%$ \\
\cline { 2 - 3 } & EVOO Sardinia & $99 \%$ \\
\cline { 2 - 3 } & EVOO Marche & $99 \%$ \\
\cline { 2 - 3 } & CVorn oil & $99 \%$ \\
\hline & EVOO Apulia & $97 \%$ \\
\hline
\end{tabular}

(a) Ratio between real matrix, corn oil, and acetone slopes of the standard addition calibration curves * 100.

\subsection{Real Samples Analyses}

After dilution or hydrolysis-and-dilution, the EVOO sample was injected via a $100 \mathrm{~nL}$ injection loop of the MS working in steady MRM conditions. The signals appearance and analysis time are only one minute for each sample. As no chromatography was involved, the samples could be injected in sequence without delay. Blanks were injected between samples, revealing no carryover.

The EVOO actual samples were produced in three different Italian regions: Marche, Apulia, and Sardinia. Different Tyr and Htyr contents were expected because the specimens come from different cultivars and soil compositions. All of the samples were diluted in acetone and hydrolyzed as described in Section 2.2. The validated method was used for the quali-quantitative analyses of Tyr and Htyr and to verify their free and total phenols content. The results obtained are reported in Table 4. The analytes' content was determined using the standard addition method described in Section 3.2.1. The free Tyr and Htyr were both below the LOQs in the Sardinia samples, whereas the free Htyr was below the LOQs in the Marche samples. The total Tyr-Htyr content spans between 32 and $44 \mathrm{mg} / \mathrm{Kg}$ for Htyr and 67 and $80 \mathrm{mg} / \mathrm{Kg}$ for Tyr in all of the samples.

Table 4. Free and total content of Tyr and Htyr in the selected oil samples. Average value of three aliquots for each EVOO sample and relative standard deviation in parentheses. b.l.: below limit of quantification. The analyte total content in $20 \mathrm{mg}$ of EVOO samples are also reported, following the 432/2012 EU Regulation.

\begin{tabular}{ccccc}
\hline \multirow{2}{*}{ Sample } & Compounds & $\begin{array}{c}\text { Free Tyr-Htyr-mg/Kg } \\
\text { (RSD\%) }\end{array}$ & $\begin{array}{c}\text { Total Tyr-Htyr-mg/Kg } \\
\text { (RSD\%) }\end{array}$ & $\begin{array}{c}\text { Total } \\
\text { Tyr-Htyr-mg/20 g } \\
\text { (RSD\%) (a) }\end{array}$ \\
\hline \multirow{2}{*}{ Apulia } & Tyr & $9(4)$ & $80(6)$ & $5(8)$ \\
& Htyr & $5(5)$ & $44(8)$ & $4(10)$ \\
\hline \multirow{2}{*}{ Marche } & Tyr & $6(7)$ & $67(9)$ & $32(4)$ \\
& Htyr & b.l. & $69(6)$ & $4(9)$ \\
\hline \multirow{2}{*}{ Sardinia } & Tyr & b.l. & $35(7)$ & \\
& Htyr & b.l. & & \\
\hline
\end{tabular}

(a) These values were calculated in accordance with the literature [35]. 
In Table 4, the results are also expressed as the absolute amount of compounds per $20 \mathrm{~g}$ of EVOOs to attest to the possible assignment of the nutraceutical label. The three oils are close to the concentration limit required to obtain the nutraceutical brand, but only Apulia oil fulfills it and could officially exhibit this claim. Although the selected EVOO samples come from regions with a rich tradition of oil, they all show poor quality, at least in their polyphenols content. None of them could bear the EU health claim based on the determination of free Tyr and Htyr.

\section{Conclusions}

Compared to other approaches recommended by the IOC, this method is simpler because it does not involve complex sample preparation procedures or chromatographic separation, only dilution before injection to determine the free analytes. Hydrolysis is necessary for the determination of the total Tyr and Htyr. The method is robust, and FIA ensures a rapid analysis of only one minute per sample. LEI-MS/MS quantitatively measures Tyr and Htyr without considerable ME, typical of other MS approaches, even with complex matrices, such as olive oil, and it does not require the support of other confirmatory techniques. The LEI-MS/MS proved to be accurate and sensitive in the range of the selected concentrations and can be used as an alternative method for the rapid screening of the free and total Tyr and Htyr content in EVOO to certify potential nutraceutical properties.

Author Contributions: Formal analysis V.T. and M.P.; writing—original draft preparation, and writing-review and editing P.P.; conceptualization and supervision A.C. and G.F. All authors have read and agreed to the published version of the manuscript.

Funding: This research did not receive any specific grant from funding agencies in the public, commercial, or not-for-profit sectors.

Acknowledgments: The authors thank Agilent Technologies for providing the LCMS/MS instrumentation.

Conflicts of Interest: The authors declare no conflict of interest.

\section{References}

1. Veneziani, G.; Sordini, B.; Taticchi, A.; Esposto, S.; Selvaggini, R.; Urbani, S.; Di Maio, I.; Servili, M. Improvement of Olive Oil Mechanical Extraction: New Technologies, Process Efficiency, and Extra Virgin Olive Oil Quality. In Products from Olive Tree; Boskou, D., Clodoveo, M.L., Eds.; Intech: Rijeka, Croatia, 2016; pp. 21-42. Available online: https: / / www.intechopen.com/books/products-from-olive-tree/improvement-of-olive-oil-mechanical-extraction-newtechnologies-process-efficiency-and-extra-virgin- (accessed on 11 February 2019). [CrossRef]

2. Obied, H.K.; Prenzler, P.D.; Omar, S.H.; Ismael, R.; Servili, M.; Esposto, S.; Taticchi, A.; Selvaggini, R.; Urbani, S. Pharmacology of olive biophenols. In Handbook of Advances in Molecular Toxicology; Fishbein, J.C., Heilman, J.M., Eds.; Elsevier: Amsterdam, The Netherlands, 2012; pp. 195-223. [CrossRef]

3. Servili, M.; Sordini, B.; Esposto, S.; Urbani, S.; Veneziani, G.; Di Maio, I.; Selvaggini, R.; Taticchi, A. Biological activities of phenolic compounds of extra virgin olive oil. Antioxidants 2014, 3, 1-23. [CrossRef]

4. Foscolou, A.; Critselis, E.; Tyrovolas, S.; Chrysohoou, C.; Sidossis, L.S.; Naumovski, N.; Matalas, A.L.; Rallidis, L.; Polychronopoulos, E.; Ayuso-Mateos, J.L.; et al. The effect of exclusive olive oil consumption on successful aging: A combined analysis of the ATTICA and MEDIS epidemiological studies. Foods 2019, 8, 25. [CrossRef] [PubMed]

5. Incani, A.; Serra, G.; Atzeri, A.; Melis, M.P.; Serreli, G.; Bandino, G.; Sedda, P.; Campus, M.; Tuberoso, C.I.; Deiana, M. Extra virgin olive oil phenolic extracts counteract the pro-oxidant effect of dietary oxidized lipids in human intestinal cells. Food Chem. Toxicol. 2016, 90, 171-180. [CrossRef]

6. Castelli, G.; Bianco, I.D.; Mizutamari, R.K. Polyphenol Content in Argentinean Commercial Extra Virgin Olive Oil. Eur. J. Lipid Sci. Technol. 2018, 120, 1800124. [CrossRef]

7. Martín-Peláez, S.; Covas, M.I.; Fitó, M.; Kušar, A.; Pravst, I. Health effects of olive oil polyphenols: Recent advances and possibilities for the use of health claims. Mol. Nutr. Food Res. 2013, 57, 760-771. [CrossRef]

8. Covas, M.I.; Nyyssönen, K.; Poulsen, H.E.; Kaikkonen, J.; Zunft, H.J.; Kiesewetter, H.; Gaddi, A.; de la Torre, R.; Mursu, J.; Bäumler, H.; et al. The effect of polyphenols in olive oil on heart disease risk factors: A randomized trial. Ann. Intern. Med. 2006, 145, 333-341. [CrossRef] [PubMed]

9. Rubió, L.; Valls, R.M.; Macià, A.; Pedret, A.; Giralt, M.; Romero, M.P.; de la Torre, R.; Covas, M.I.; Solà, R.; Motilva, M.J. Impact of olive oil phenolic concentration on human plasmatic phenolic metabolites. Food Chem. 2012, 135, 2922-2929. [CrossRef] [PubMed] 
10. Estruch, R.; Martinez-Gonzalez, M.A.; Corella, D.; Salas-Salvado, J.; Ruiz-Gutierrez, V.; Covas, M.I.; Fiol, M.; Gómez-Garcia, E.; López-Sabater, M.C.; Vinyoles, E.; et al. Effects of a Mediterranean-style diet on cardiovascular risk factors: A randomized trial. Ann. Intern. Med. 2006, 145, 1-11. [CrossRef]

11. Goncalves, I.; Andersson-Georgiadou, E.; Mattsson, S.; Skog, G.; Pedro, L.M.; Fernandes e Fernandes, J.; Dias, N.; Engström, G.; Nilsson, J.; Stenström, K. Direct association between diet and the stability of human atherosclerotic plaque. Sci. Rep. 2015, 5, 15524. [CrossRef]

12. Couto, E.; Boffetta, P.; Lagiou, P.; Ferrari, P.; Buckland, G.; Overvad, K.; Dahm, C.C.; Tjønneland, A.; Olsen, A.; Clavel-Chapelon, F.; et al. Mediterranean dietary pattern and cancer risk in the EPIC cohort. Br. J. Cancer 2011, 104, 1493-1499. [CrossRef]

13. Gonzalez-Alonso, A.; Perez-Lopez, P.; Varela-Lopez, A.; Ramirez-Tortosa, M.C.; Battino, M.; Quiles, J.L. Experimental evidence on the role of different types unsaturated fats in the diet on ageing. Rev. Esp. Geriatr. Gerontol. 2015, 50, $285-288$.

14. Varela-Lopez, A.; Bullon, P.; Battino, M.; Ramirez-Tortosa, M.; Ochoa, J.J.; Cordero, M.D.; Ramirez-Tortosa, C.L.; Rubini, C.; Zizzi, A.; Quiles, J.L. Coenzyme Q protects against age-related alveolar bone loss associated to n-6 polyunsaturated fatty acid rich-diets by modulating mitochondrial mechanisms. J. Gerontol. A Biol. Sci. Med. Sci. 2015, 71, 593-600. [CrossRef]

15. Visioli, F.; Franco, M.; Toledo, E.; Luchsinger, J.; Willett, W.C.; Hu, F.B.; Martinez-Gonzalez, M.A. Olive oil and prevention of chronic diseases: Summary of an International conference. Nutr. Metab. Cardiovasc. Dis. 2018, 28, 649-656. [CrossRef]

16. Robles-Almazana, M.; Pulido-Moran, M.; Moreno-Fernandez, J.; Ramirez-Tortosa, C.; Rodriguez-Garcia, C.; Quiles, J.L.; Ramirez-Tortosa, M.C. Hydroxytyrosol: Bioavailability, toxicity, and clinical applications. Food Res. Int. 2018, 105, 654-667. [CrossRef] [PubMed]

17. Vilaplana-Pérez, C.; Auñón, D.; García-Flores, L.A.; Gil-Izquierdo, A. Hydroxytyrosol and potential uses in cardiovascular diseases, cancer, and AIDS. Front. Nutr. 2014, 1, 18. [CrossRef]

18. European Commission. Regulation EC No. 432/2012 establishing a list of permitted health claims made on foods, other than those referring to the reduction of disease risk and to children's development and health. Off. J. Eur. Union 2012, L136, 1-40. Available online: https:/ / eurlex.europa.eu/legalcontent/GA/TXT/?uri=CELEX:32012R0432 (accessed on 11 February 2019).

19. Pichierri, M.; Peluso, A.M.; Pino, G.; Guido, G. Communicating the health value of extra-virgin olive oil: An investigation of consumers' responses to health claims. Br. Food J. 2021, 123, 492-508. [CrossRef]

20. International Olive Council. Determination of Biophenols in Olive Oils by HPLC. 2009 COI/T.20/Doc.No 29/1-8. Available online: https: / / www.oelea.de/downloads/COI-T20-DOC-29-2009-DETERMINATION-OF-BIOPHENOLS-IN-OLIVE-OILSBY-HPLC.pdf (accessed on 15 February 2019).

21. Nenadis, N.; Mastralexi, A.; Tsimidou, M.Z.; Vichi, S.; Quintanilla-Casas, B.; Donarski, J.; Bailey-Horne, V.; Butinar, B.; Miklavčič, M.; García González, D.L.; et al. Toward a harmonized and standardized protocol for the determination of total hydroxytyrosol and tyrosol content in virgin olive oil (VOO). Extraction solvent. Eur. J. Lipid Sci. Technol. 2018, 120, 1800099. [CrossRef]

22. Tsimidou, M.Z.; Sotiroglou, M.; Mastralexi, A.; Nenadis, N.; García-González, D.L.; Toschi, T.G. In house validated UHPLC protocol for the determination of the total hydroxytyrosol and tyrosol content in virgin olive oil fit for the purpose of the health claim introduced by the EC regulation 432/2012 for "olive oil polyphenols. Molecules 2019, 24, 1044. [CrossRef]

23. Ricciutelli, M.; Marconi, S.; Boarelli, M.C.; Caprioli, G.; Sagratini, G.; Ballini, R.; Fiorini, D. Olive oil polyphenols: A quantitative method by high-performance liquid-chromatography-diode-array detection for their determination and the assessment of the related health claim. J. Chromatogr. A 2017, 1481, 53-63. [CrossRef]

24. Purcaro, G.; Codony, R.; Pizzale, L.; Mariani, C.; Conte, L. Evaluation of total hydroxytyrosol and tyrosol in extra virgin olive oils. Eur. J. Lipid Sci. Technol. 2014, 116, 805-811. [CrossRef]

25. Hashemi, P.; Serenjeh, F.N.; Ghiasvand, A.R. Reversed-phase dispersive liquid-liquid microextraction with multivariate optimization for sensitive HPLC determination of tyrosol and hydroxytyrosol in olive oil. Anal. Sci. 2011, 27, 943. [CrossRef] [PubMed]

26. Romero, C.; Brenes, M. Analysis of total contents of hydroxytyrosol and tyrosol in olive oils. J. Agric. Food Chem. 2012, 60, 9017-9022. [CrossRef]

27. Bellumori, M.; Cecchi, L.; Innocenti, M.; Clodoveo, M.L.; Corbo, F.; Mulinacci, N. The EFSA health claim on olive oil polyphenols: Acid hydrolysis validation and total hydroxytyrosol and tyrosol determination in italian virgin olive oils. Molecules 2019, $24,2179$. [CrossRef]

28. Olmo-García, L.; Fernández-Fernández, C.; Hidalgo, A.; Vílchez, P.; Fernández-Gutiérrez, A.; Marchal, R.; Carrasco-Pancorbo, A. Evaluating the reliability of specific and global methods to assess the phenolic content of virgin olive oil: Do they drive to equivalent results? J. Chromatogr. A 2018, 1585, 56-69. [CrossRef] [PubMed]

29. Mohamed, M.B.; Guasmi, F.; Ali, S.B.; Radhouani, F.; Faghim, J.; Triki, T.; Kammoun, N.G.; Baffi, C.; Lucini, L.; Benincasa, C. The LC-MS/MS characterization of phenolic compounds in leaves allows classifying olive cultivars grown in South Tunisia. Biochem. Syst. Ecol. 2018, 78, 84-90. [CrossRef]

30. Bartella, L.; Mazzotti, F.; Napoli, A.; Sindona, G.; Di Donna, L. A comprehensive evaluation of tyrosol and hydroxytyrosol derivatives in extra virgin olive oil by microwave-assisted hydrolysis and HPLC-MS/MS. Anal. Bioanal. Chem. 2018, 410, 2193-2201. [CrossRef] 
31. Monasterio, R.P.; Olmo-García, L.; Bajoub, A.; Fernández-Gutiérrez, A.; Carrasco-Pancorbo, A. phenolic compounds profiling of virgin olive oils from different varieties cultivated in mendoza, argentina, by using liquid chromatography-mass spectrometry. J. Agric. Food Chem. 2017, 65, 8184-8195. [CrossRef]

32. Mazzotti, F.; Benabdelkamel, H.; Di Donna, L.; Maiuolo, L.; Napoli, A.; Sindona, G. Assay of tyrosol and hydroxytyrosol in olive oil by tandem mass spectrometry and isotope dilution method. Food Chem. 2012, 135, 1006-1010. [CrossRef]

33. Bazoti, F.N.; Gikas, E.; Skaltsounis, A.L.; Tsarbopoulos, A. Development of a liquid chromatography-electrospray ionization tandem mass spectrometry (LC-ESI MS/MS) method for the quantification of bioactive substances present in olive oil mill wastewaters. Anal. Chim. Acta 2006, 573-574, 258-266. [CrossRef]

34. Conte, L.; Bendini, A.; Valli, E.; Lucci, P.; Moret, S.; Maquet, A.; Lacoste, F.; Brereton, P.; García-González, D.L.; Moreda, W.; et al. Olive oil quality and authenticity: A review of current EU legislation, standards, relevant methods of analyses, their drawbacks and recommendations for the future. Trends Food Sci. Technol. 2020, 105, 483-493. [CrossRef]

35. Bartella, L.; Mazzotti, F.; Sindona, G.; Napoli, A.; Di Donna, L. Rapid determination of the free and total hydroxytyrosol and tyrosol content in extra virgin olive oil by stable isotope dilution analysis and paper spray tandem mass spectrometry. Food Chem. Toxicol. 2020, 136, 111110. [CrossRef] [PubMed]

36. Luque-Muñoz, A.; Tapia, R.; Haidour, A.; Justicia, J.; Cuerva, J.M. Direct determination of phenolic secoiridoids in olive oil by ultra-high performance liquid chromatography-triple quadruple mass spectrometry analysis. Sci. Rep. 2019, 9, 15545. [CrossRef]

37. Termopoli, V.; Famiglini, G.; Palma, P.; Piergiovanni, M.; Cappiello, A. Atmospheric pressure vaporization mechanism for coupling a liquid phase with electron ionization mass spectrometry. Anal. Chem. 2017, 89, 2049-2056. [CrossRef] [PubMed]

38. Termopoli, V.; Famiglini, G.; Palma, P.; Piergiovanni, M.; Rocio-Bautista, P.; Ottaviani, M.F.; Cappiello, A.; Saeed, M.; Perry, S. Evaluation of a liquid electron ionization liquid chromatography-mass spectrometry interface. J. Chromatogr. A 2019, 1591, 120-130. [CrossRef] [PubMed]

39. European Commission. 2002/657/EC: Commission decision of 12 august 2002 implementing council directive 96/23/EC concerning the performance of analytical methods and the interpretation of results. Off. J. Eur. Commun. 2002, L221, 8-36.

40. Rocío-Bautista, P.; Famiglini, G.; Termopoli, V.; Palma, P.; Nazdrajić, E.; Pawliszyn, J.; Cappiello, A. Direct coupling of bio-SPME to liquid electron ionization-MS/MS via a modified microfluidic open interface. J. Am. Soc. Mass Spectrom. 2021, 32, 262-269. [CrossRef] [PubMed] 